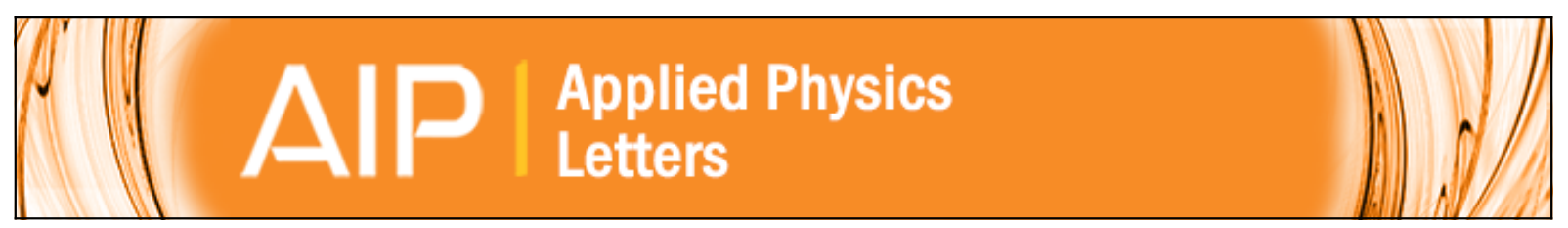

\title{
Nonlinear focusing of ultrasonic waves by an axisymmetric diffraction grating embedded in water
}

N. Jiménez, V. Romero-García, R. Picó, L. M. Garcia-Raffi, and K. Staliunas

Citation: Applied Physics Letters 107, 204103 (2015); doi: 10.1063/1.4935917

View online: http://dx.doi.org/10.1063/1.4935917

View Table of Contents: http://scitation.aip.org/content/aip/journal/apl/107/20?ver=pdfcov

Published by the AIP Publishing

\section{Articles you may be interested in}

Quantitative measurement of phase variation amplitude of ultrasonic diffraction grating based on diffraction spectral analysis

Rev. Sci. Instrum. 85, 093112 (2014); 10.1063/1.4895649

Measurement of irradiation effects in precipitate hardened aluminum using nonlinear ultrasonic principles (in-situ) AIP Conf. Proc. 1430, 1648 (2012); 10.1063/1.4716411

On The Behavior Of Nonlinear Ultrasonic Waves In Water-air Mixtures

AIP Conf. Proc. 1022, 73 (2008); 10.1063/1.2956305

Old World frog and bird vocalizations contain prominent ultrasonic harmonics

J. Acoust. Soc. Am. 115, 910 (2004); 10.1121/1.1636851

Second harmonic component in focused ultrasound transmitted through a solid plate

AIP Conf. Proc. 524, 199 (2000); 10.1063/1.1309204

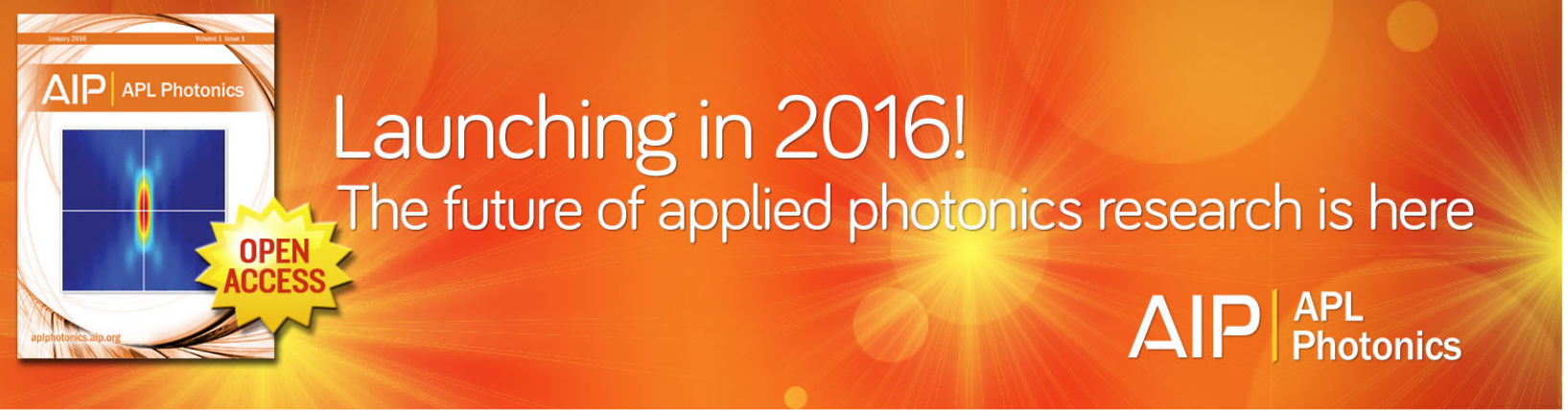




\title{
Nonlinear focusing of ultrasonic waves by an axisymmetric diffraction grating embedded in water
}

\author{
N. Jiménez, ${ }^{1}$ V. Romero-García, ${ }^{2}$ R. Picó, ${ }^{1}$ L. M. Garcia-Raffi, ${ }^{3}$ and K. Staliunas ${ }^{4}$ \\ ${ }^{1}$ Instituto de Investigación para la Gestión Integrada de zonas Costeras, Universitat Politècnica de València, \\ Paranimf 1, 46730 Grao de Gandia, València, Spain \\ ${ }^{2}$ LUNAM Université, Université du Maine, LAUM UMR CNRS 6613, Av. O. Messiaen, 72085 Le Mans, France \\ ${ }^{3}$ Instituto Universitario de Matemática Pura y Aplicada, Universitat Politècnica de València, Camino de Vera \\ s/n, 46022 València, Spain \\ ${ }^{4}$ ICREA, Departament de Física i Enginyeria Nuclear, Universitat Politècnica de Catalunya, Colom, 11, \\ E-08222 Terrassa, Barcelona, Spain
}

(Received 26 July 2015; accepted 4 November 2015; published online 16 November 2015)

\begin{abstract}
We report the nonlinear focusing of ultrasonic waves by an axisymmetric diffraction grating immersed in water. In the linear regime, the system presents high focal gain $(32 \mathrm{~dB})$, with a narrow beam-width and intense side lobes as it is common in focusing by Fresnel-like lenses. Activating the nonlinearity of the host medium by using high amplitude incident waves, the focusing properties of the lens dramatically change. Theoretical predictions show that the focal gain of the system extraordinary increases in the strongly nonlinear regime (Mach number of $6.1 \times 10^{-4}$ ). Particularly, the harmonic generation is locally activated at the focal spot, and the second harmonic beam is characterized by strongly reduced side-lobes and an excellent beam profile as experiments show in agreement with theory. The results can motivate applications in medical therapy or second harmonic imaging. (C) 2015 AIP Publishing LLC. [http://dx.doi.org/10.1063/1.4935917]
\end{abstract}

Acoustic focusing is an interdisciplinary topic covering a rich set of applied sciences as, for example, nondestructive testing techniques, detection of fish and undersea exploration, therapeutic techniques, diagnostic sonography, and ultrasound surgery in medical applications among others. In the most common cases, acoustic lenses are constructed by a refractive material with curved interfaces analogously to the optical lenses. Flat diffraction grating plates have been proposed as acoustic analogues of the Fresnel Zone Plates (FZPs) ${ }^{1-4}$ to produce acoustic focusing. Acoustic FZPs consist of alternating rigid (opaque) and open (transparent) annuli with the radius and width specially designed to produce constructive interference at focus. This kind of diffraction gratings have been used to design bi-focal lenses ${ }^{5}$ or to enhance the acoustic transmission. ${ }^{4}$ Recently, acoustic diffraction gratings based on discrete axisymmetric arrays of toroidal scatterers have been proposed to generate efficient acoustic Bessel-like beams. ${ }^{6}$ In all of these cases, focusing can result in high intensities at a specific location over a small volume, triggering in most of the cases nonlinear acoustic phenomena, and enhancing specific mechanical and thermal effects. However, the nonlinear focusing using diffraction gratings is a less explored area.

In general, mechanical phenomena include acoustic streaming, radiation forces, ${ }^{7-9}$ and cavitation, ${ }^{10,11}$ while the nonlinear thermal effects include heat generation due to nonlinear absorption of the acoustic energy. These nonlinear effects are exploited in a broad series of applications as, for example, harmonic imaging techniques ${ }^{12,13}$ or medical therapy. ${ }^{11}$ Therefore, the design of devices that easily manage the focusing properties and the enhancement of the focal gain is of pivotal importance.

In spherical focusing devices [see Fig. 1(a)], the gain is related to the aperture of the system in such a way that the

maximum gain is obtained with an aperture equal to the focal distance, ${ }^{14}$ being very difficult the focusing inside a target medium with high gains. In this work, we present the transition from the linear- to the nonlinear-focusing of ultrasonic waves by an axisymmetric grating made of rigid toroidal scatterers embedded in water. In the linear regime, we show that the device presents a narrow focal spot, which is between the diffraction and the sub-wavelength limits with high focal gain $(32 \mathrm{~dB})$. As common in Fresnel-like lenses and also for Bessel-like beams, ${ }^{6,15}$ the narrow focal spot is accompanied by high amplitude circular side-lobes. By increasing the amplitude of the incident waves, the second order nonlinear effects of water become important. We numerically analyze the transition from the linear to the nonlinear regime of the lens. As the amplitude of the incident wave increases, the focal gain dramatically increases in comparison to the linear regime, reaching values around $40 \mathrm{~dB}$. Moreover, due to the strong enhancement of the field at

(a)

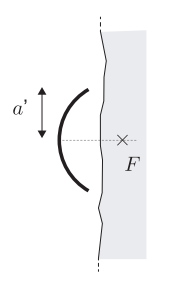

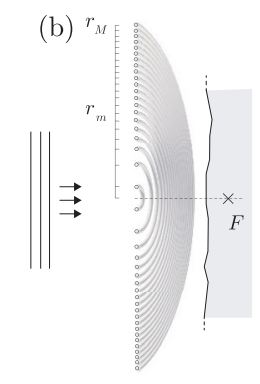

FIG. 1. (a) Equivalent passive spherical focusing device. The design depicts a lens with an aperture, $A=2 a^{\prime}=F$, to have the focal located inside the target medium, as achieved by the axisymmetric grating analyzed in this work. (b) Scheme of the axisymmetric lens. (c) Photograph of the experimental device shown in the inset. 
focus, the higher harmonic generation is activated around the focal spot. These local nonlinear processes produce higher harmonics that maintain the narrow beam-like in the linear regime while its axial- and side-lobes are strongly reduced. In the current work, we demonstrate these results by analytical estimations, numerical simulations and experiments. A simple analytical model based on an approach of axisymmetric diffraction gratings is used to estimate the focal positions and the size of the focal spot. Numerical calculations (using finite-difference time-domain (FDTD) techniques ${ }^{16}$ ) of acoustic waves propagating through such axisymmetric gratings were used to calculate the complete acoustic field in both the linear and the nonlinear regimes. The experimental verification of the theoretical prediction is also reported in both the linear and the weakly nonlinear regime showing good agreement with the theory.

We consider $M$ concentric toroidal scatterers of minor radius $R$ and major radius $r_{m}$ with $m=1, \ldots, M$ being the $z$ direction along the symmetry axis of the system (see Fig. 1(b)). A plane wave with amplitude $p_{0}$ impinges the axisymmetric grating from the negative to the positive $z$ direction. We consider that $R$ is small in comparison to the incident wavelength, $R \ll \lambda$. In order to have an optimal superposition at a given focal distance, $F$, behind the axisymmetric grating, all the incoming waves should arrive precisely in phase to $F$. This suggests an ideal axisymmetric grating would consist of a series of infinitesimal toroidal scatterers located in a plane at radial distances given by

$$
r_{m+1}^{2}=r_{m}^{2}+n \lambda\left(n \lambda+2 \sqrt{r_{m}^{2}+F^{2}}\right),
$$

where $n$ indicates the diffraction order of the axisymmetric diffraction grating. Equivalently, for a given distribution of toroidal scatterers, $r_{m}$, the focal distance can be determined from two consecutive positions in the grating by the following expression:

$$
F=\sqrt{\left(\frac{r_{m+1}^{2}-r_{m}^{2}}{2 n \lambda}-\frac{n \lambda}{2}\right)^{2}-r_{m}^{2}} .
$$

The axisymmetric grating is designed for a focal distance of $F=15 \mathrm{~mm}$ and frequency $f_{0}=c / \lambda_{0}=2.229 \mathrm{MHz}$ for the first diffraction order $(n=1)$. The grating is fabricated by laser cutting over a stainless steel plate of $0.8 \mathrm{~mm}$ thickness. The minor radius of the toroidal scatterers is $R=0.75 \mathrm{~mm}$ (solid parts). The number of rings was $M=21$ and the radius of the outer ring was $r_{M}=25 \mathrm{~mm}$. The axisymmetric grating lens was placed in front of an ultrasound plane transducer (see Fig. 1(c)). This source was formed by a plane single element piezoceramic element mounted in a custom designed steel housing with $50 \mathrm{~mm}$ of aperture, matching the area of the grating. The separation distance between the grating and the source plane was adjusted to $0.5 \mathrm{~mm}$. The assembled grating-transducer system was immersed in a degassed and distilled water tank of $350 \times 350 \times 350 \mathrm{~mm}^{3}$, the temperature of which during the measurements was constant at $26^{\circ} \mathrm{C}$. The pressure waveforms were acquired with an $\mathrm{HNR}$ $500 \mu$ m needle PVDF hydrophone (Onda Corp., CA), moved by a three-axis micropositioning system (OWIS GmbH, Germany) with an accuracy of $10 \mu \mathrm{m}$.
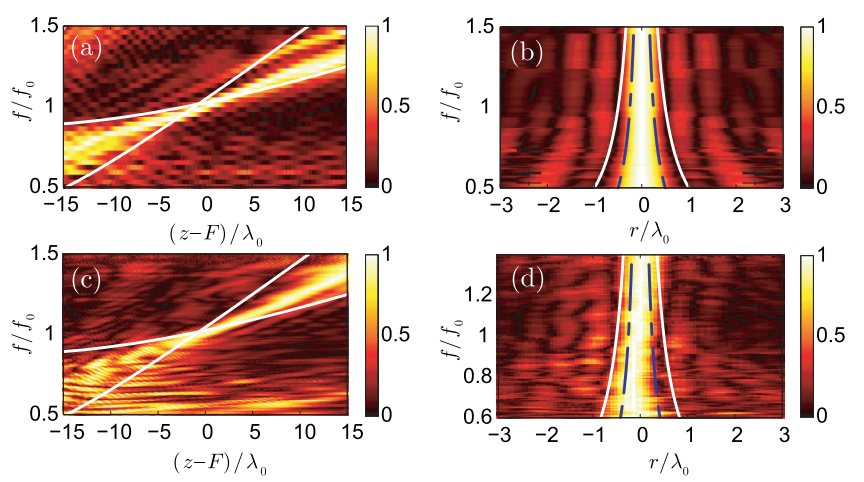

FIG. 2. Characterization of the linear focusing properties of the axisymmetric diffraction grating. (a) and (b) The numerical results of the acoustic field along the axis of symmetry (longitudinal direction) of the system $(r / \lambda=0)$ and across the radial direction in the position of the focus, $(z-F) / \lambda=0$ in terms of normalized frequency. (c) and (d) The corresponding experimental results. In (a)-(d), the color map represents the pressure field normalized to the maximum at each frequency. White lines in (a) and (c) represent the analytical predictions of the focusing position. White dashed and blue dotdashed lines in (b) and (d) represent the sub-wavelength $(\mathrm{FWHM}<\lambda$ ) and diffraction $(\mathrm{FWHM}<\lambda / 2)$ limits respectively.

We start from the theoretical and experimental results of the linear behavior of the system. Figures 2(a) and 2(b) show the focal properties calculated for the first diffraction grating mode, i.e., $n=1$. In Fig. 2(a), the normalized pressure field along the symmetry axis in terms of frequency is shown. The white dotted lines represent the curves obtained from Eq. (2) for two different pairs of $m(m=1,2$ and $m=20,21)$, the crossing of these lines represents the focal point. Although the lens is designed to focus waves at focal distance $F$ and at frequency $f_{0}$, the focal spot presents weak chromatic aberration giving rise to the possibility to tune the position of the focus with frequency. ${ }^{14}$ Figure 2 (b) represents the radial distribution of the normalized acoustic field at the position of the focal spot. We observe a main lobe aligned with the axis of the axisymmetric lens with secondary smaller lobes. Figures 2(c) and 2(d) represent the equivalent experimental results to Figs. 2(a) and 2(b) respectively.

We now evaluate the focusing capability of the axisymmetric grating, by analyzing both the focal gain and the full width at half maximum (FWHM) of the focus. The focal gain is evaluated using the expression $G[\mathrm{~dB}]=20 \log \left(\left|p_{\max }\right| /\left|p_{0}\right|\right)$, where $p_{\max }$ is the pressure at the focal point. For this lens, the focal gain value is $32 \mathrm{~dB}\left(\left|p_{\max }\right| /\left|p_{0}\right|=40\right)$ at $f \mid f_{0}=1$ (experimental measured gain of $30 \mathrm{~dB}$ ). The resolution of the focus is characterized by $\lambda / 2<$ FWHM $<\lambda$ for the whole range of analyzed frequencies. This results in a sub-wavelength FWHM close to the diffraction limit.

Next, we numerically analyze the transition to the nonlinear-focusing. The source of nonlinearity is considered in the water (parameter of nonlinearity is $\beta=3.5$, density $\rho_{0}=1000 \mathrm{~kg} / \mathrm{m}^{3}$, and sound speed $c_{0}=1500 \mathrm{~m} / \mathrm{s}^{17}$ ). In order to activate the nonlinearity of the medium, the amplitude of the incident waves is increased. These amplitudes are expressed here using the normalized quantity $F / x_{s}$, being $x_{s}=1 / k \epsilon \beta$ the shock formation distance with $k$ the wavenumber and $\epsilon=p_{0} / \rho_{0} c_{0}^{2}$ the acoustic Mach number. ${ }^{17}$ Figures 3(a) and 3(b), respectively, show the longitudinal and radial pressure distribution at the focus for different excitation amplitudes. The small-amplitude pressure-distribution of a 

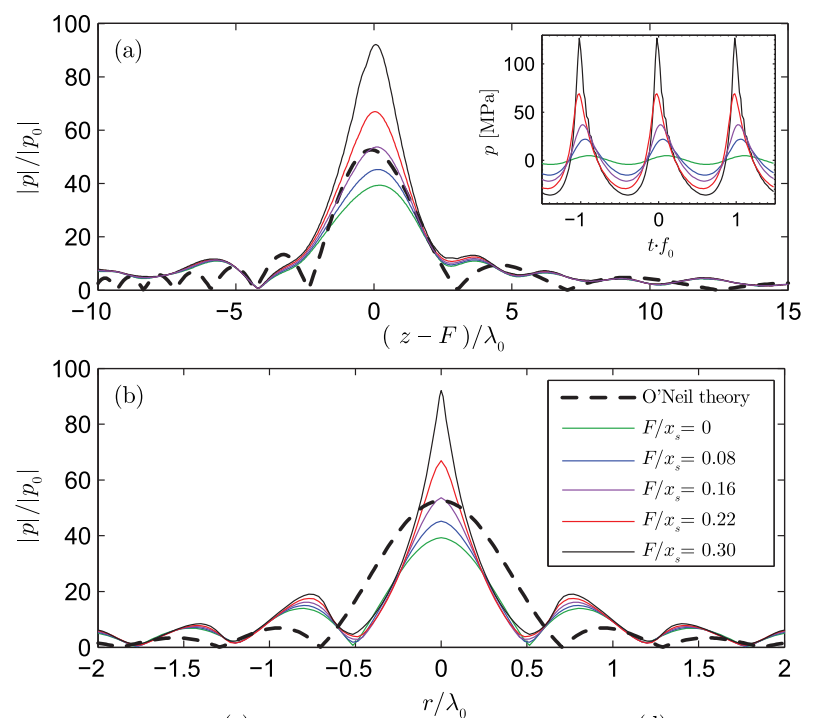

(c)
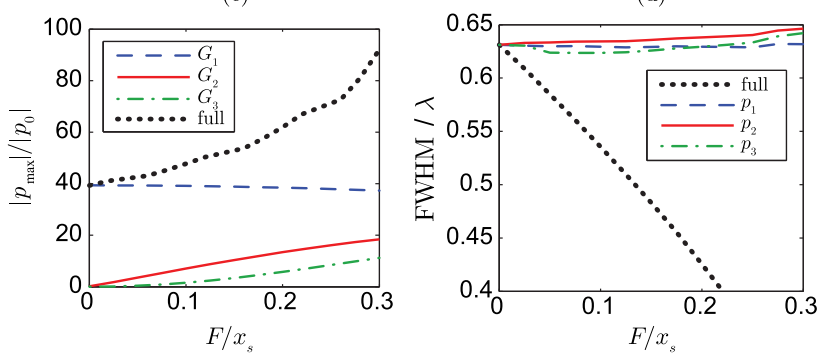

FIG. 3. Numerical characterization of the nonlinear focusing properties of the axisymmetric diffraction grating. (a) and (b) The longitudinal and radial pressure profiles calculated for different excitation amplitudes. The field of an equivalent spherically focused device is shown in dashed black lines for comparison. Inset in (a) shows the waveforms at focus for each amplitude. (c) and (d)The numerical analysis of the dependence of the focal gain and of the full width at half maximum (FWHM) on the amplitude of the incident wave, evaluated in terms of the normalized amplitude, $F / x_{s}$, for the first (blue dashed line), second (red continuous line), third (green dotted line) harmonics and the full wave (black dotted line).

passive spherical radiator (see Fig. 1(a)) with same parameters is also shown for comparison in dotted lines. Although the spherical radiator gain is comparable to the gain obtained with the grating lens, the FWHM of the beam is strongly reduced in the focus produced by the grating. Furthermore, the effective focal gain of the system (the peak value) is extraordinary increased from the linear value of $32 \mathrm{~dB}$ to $40 \mathrm{~dB}\left(\left|p_{\max }\right| /\left|p_{0}\right|=96\right)$ in the case of strong nonlinearity $\left(\epsilon=6.1 \times 10^{-4}\right)$. Especially, the numerical results show that the waveform is dramatically distorted around the focal spot, while it is stable out of the focal region. As the peak pressure grows locally at the focus, the focal width stretches in both axial and lateral directions around the focal spot. This means that the nonlinearity is activated mostly locally at the focal spot and the higher harmonics can be generated in this region. In addition, the ratio between the peak pressure and its lateral lobes is strongly increased. This gives unique spatial properties for the higher harmonics that preserve the narrow beam-width of the Fresnel lens, while its axial- and side-lobes are strongly reduced.

Now, we analyze the local harmonic generation. Figure 3(c) shows the focal gain of the different harmonics as the amplitude of the incident wave is increased ( $F / x_{s}$ increased). It is worth noting here that for low amplitudes of the incident wave, we recover the results discussed earlier for the linear regime. The focal gain for the first harmonic is almost constant, around $32 \mathrm{~dB}\left(\left|p_{\max }\right| /\left|p_{0}\right|=40\right)$, but it slightly reduces for increasing amplitudes as energy is transferred to higher harmonics, being the total energy conserved. Particularly, the focal gain for second and higher harmonics increases. In the case of second harmonic it varies from 0 in the linear regime to about the half of the fundamental first harmonic amplitude.

The dependence of FWHM on the incident wave amplitude is shown in Fig. 3(d). FWHM remains constant for both, fundamental and second harmonic focal spots. Note here that the second harmonic focal size is approximately half of the focal size of the fundamental wave, since we show the value normalized to its wavelength. It is worth noting here, that as the nonlinearity of the medium is activated the FWHM of the focal spot considering all the harmonics dramatically decreases.

At this stage, we experimentally test the case $F / x_{s}=0.02$ $\left(\epsilon=4 \times 10^{-5}\right)$ in order to evaluate the locally generated higher harmonic and their spatial profiles. This situation corresponds to the weakly nonlinear regime in which our experimental set-up can be used with a linear electro-acoustic response in the range of frequencies from $0.5 f / f_{0}$ to $1.5 f / f_{0}$ ensuring that the observed nonlinear effects come only from the nonlinearity of water (see insets of Figs. 4(b) and 4(c)). In this range of frequencies, as shown in Fig. 4(a), we experimentally and numerically reproduce similar acoustic pattern as in the linear regime, showing a focal spot at $f / f_{0}=1$ and at
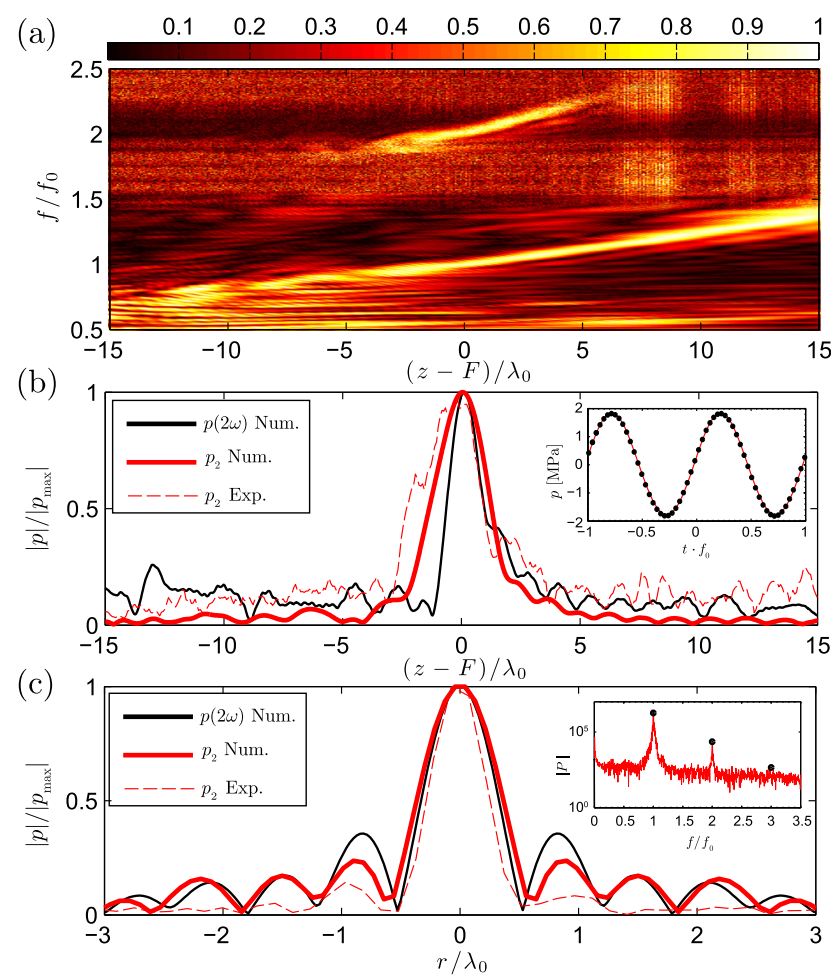

FIG. 4. (a) Experimental evidence of the generation of the second harmonic at the focal position in the weakly nonlinear regime $\left[F / x_{s}=0.02\right.$ $\left.\left(\epsilon=4 \times 10^{-5}\right)\right]$. Color scale shows the normalized pressure with respect to the maximum at each frequency. (b) and (c) The comparison of the longitudinal and radial profiles for the second harmonic nonlinearly generated, $p_{2}$, with the linearly generated, $p_{2 \omega}$. Insets in (b) and (c) show the corresponding waveforms and spectra at focus respectively. 
focal distance $z=F$. However, now we can clearly see the presence of the second harmonic at the focal spot, i.e., at double frequency $f / f_{0}=2$. As discussed above, due to the nonlinearity of water the high amplitude around the focal spot at frequency $f / f_{0}=1$ activates the harmonic generation, producing a focal spot at $f / f_{0}=2$ (experimental gain of $31 \mathrm{~dB})$.

Figures 4(b) and 4(c) show the comparison between longitudinal and radial profiles of the second harmonic, $p_{2}$, and the profiles obtained at frequency $2 \omega, p_{2 \omega}$, in the linear regime, respectively. Red continuous and red dashed lines represent the numerical and experimental profiles of the second harmonic, $p_{2}$, while black continuous lines represent the profiles of the linear focusing at frequency $2 \omega, p_{2 \omega}$. The differences between the numerical and experimental results for the longitudinal profiles come from the reflections produced in the cavity between the axisymmetric lens and the transducer. Profiles from nonlinear generation present a similar lateral spatial resolution but slightly bigger depth focus than in the linear regime. This is because the first harmonic is the acoustic source of the second one. Moreover, the nonlinear profiles present smaller axial- and side-lobes. Specifically, we report a side-lobe reduction of $54 \%$ in experiment $(33 \%$ numerical) relative to the fundamental harmonic side-lobe amplitude.

We have presented the nonlinear characterization of the focusing properties of an axisymmetric ultrasonic diffraction grating constructed by toroidal rigid scatters embedded in water. Theory and experiments are in good agreement in both the linear and the nonlinear regime. The confined focal spot provides strong energy concentration over a small volume to activate the nonlinear effects locally at the focal spot, giving rise to an almost local higher harmonic generation. The field of the higher harmonics maintains the excellent beam-width of the Fresnel lens, while its axial- and sidelobes are strongly reduced. This effect is remarkable even in the weakly nonlinear regime, and can be used to improve the image quality by the well-known harmonic imaging method $^{12,13}$ in which images are created from second (or higher) harmonic signals. Therefore, the presented passive system can be suitable for second harmonic imaging techniques. It can also motivate applications in medical therapy. ${ }^{17}$

The work was supported by Spanish Ministry of Science and Innovation and European Union FEDER through Projects FIS2011-29734-C02-01 and -02, MTM2012-36740c02-02, and PAID 2012/253.

${ }^{1}$ S. Farnow and B. Auld, Appl. Phys. Lett. 25, 681 (1974).

${ }^{2}$ M. Z. Sleva, W. D. Hunt, and R. D. Briggs, J. Acoust. Soc. Am. 96, 1627 (1994).

${ }^{3}$ H. Wang, D. Xing, and L. Xiang, J. Phys. D: Appl. Phys. 41, 095111 (2008).

${ }^{4}$ M. Molerón, M. Serra-Garcia, and C. Daraio, Appl. Phys. Lett. 105, 114109 (2014).

${ }^{5}$ G. Clement, H. Nomura, and T. Kamakura, IEEE Trans. Ultrason. Ferroelectr. Freq. Control 62, 350 (2015).

${ }^{6}$ N. Jiménez, V. Romero-García, R. Picó, A. Cebrecos, V. SánchezMorcillo, L. Garcia-Raffi, J. Sánchez-Pérez, and K. Staliunas, Europhys. Lett. 106, 24005 (2014).

${ }^{7}$ A. P. Sarvazyan, O. V. Rudenko, S. D. Swanson, J. B. Fowlkes, and S. Y. Emelianov, Ultrasound Med. Biol. 24, 1419 (1998).

${ }^{8}$ K. Nightingale, M. S. Soo, R. Nightingale, and G. Trahey, Ultrasound Med. Biol. 28, 227 (2002).

${ }^{9}$ E. E. Konofagou and K. Hynynen, Ultrasound Med. Biol. 29, 1405 (2003).

${ }^{10}$ F. A. Duck, Ultrasound Med. Biol. 28, 1 (2002).

${ }^{11}$ M. Bailey, V. Khokhlova, O. Sapozhnikov, S. Kargl, and L. Crum, Acoust. Phys. 49, 369 (2003).

${ }^{12}$ M. A. Averkiou, D. N. Roundhill, and J. Powers, in IEEE Proceedings of the Ultrasonics Symposium (IEEE, 1997), Vol. 2, pp. 1561-1566.

${ }^{13}$ M. M. Nguyen, J. Shin, and J. Yen, Ultrasound Med. Biol. 40, 2488 (2014).

${ }^{14}$ See supplementary material at http://dx.doi.org/10.1063/1.4935917 for additional comparisons of the focusing properties of the axisymmetric lens presented in this work with the classical spherical radiators. Moreover, additional experimental results show the weak chromatic aberration of the axisymmetric lens (see also the accompanying video).

${ }^{15}$ J.-y. Lu and J. Greenleaf, IEEE Trans. Ultrason. Ferroelectr. Freq. Control 37, 438 (1990).

${ }^{16}$ N. Jiménez, "Nonlinear acoustic waves in complex media," Ph.D. thesis, Universitat Politècnica de València, 2015.

${ }^{17}$ Nonlinear Acoustics, edited by M. Hamilton and D. Blackstock (Academic Press, San Diego, CA 92101, USA, 1998). 